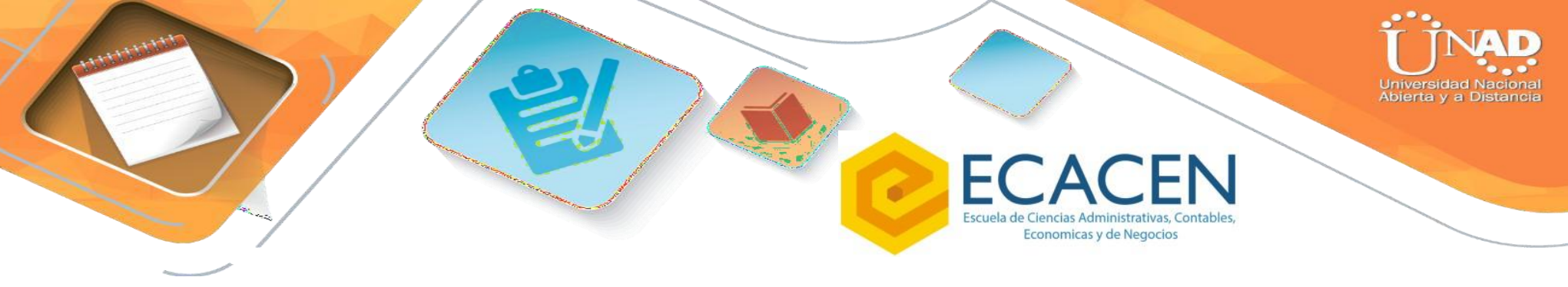

\title{
REVOLUCIÓN 4.0: NUEVAS COMPETENCIAS REQUERIDAS EN ESTUDIANTES DE EDUCACIÓN SUPERIOR PARA MIGRAR A LA EDUCACIÓN 4.0
}

\section{REVOLUCIÓN 4.0: NUEVAS COMPETENCIAS REQUERIDAS EN ESTUDIANTES DE EDUCACIÓN SUPERIOR PARA MIGRAR A LA EDUCACIÓN 4.0}

\author{
Autor 1 \\ Zuyin Ch. Lima Hernández \\ Estudiante de Administración de empresas \\ Escuela de Ciencias Administrativas, contables, Económicas y de \\ negocios \\ Universidad Nacional Abierta y a Distancia UNAD - Cartagena \\ ORCID: https://orcid.org/0000-0003-0552-3778
}

\section{Resumen}

El presente es un informe parcial del estudio sobre las competencias que debe tener los estudiantes de educación superior para migrar a la educación 4.0, a través de este se hace un primer acercamiento teórico a la realidad de la revolución 4.0, las características de las organizaciones inteligentes y la evolución que debe tener la educación superior para formar profesionales integrales para las nuevas demandas del mercado laboral. Se presenta un marco teórico general sobre cómo se da en esencia la revolución 4.0, y cómo esto debe afectar la educación tradicional para evolucionar a nuevos paradigmas, generando nuevas demandas para el docente y su práctica pedagógica, esencialmente para el estudiante universitario y su autonomía para aprender de manera flexible pero significativa.

Palabras clave: educación 4.0, industria 4.0, organizaciones inteligentes, revolución 4.0

\begin{abstract}
This is a partial report of the study on the competencies that higher education students should have to migrate to education 4.0, through this a first theoretical approach to the reality of the 4.0 revolution, the characteristics of intelligent organizations and the evolution that higher education should have to train integral professionals for the new demands
\end{abstract}




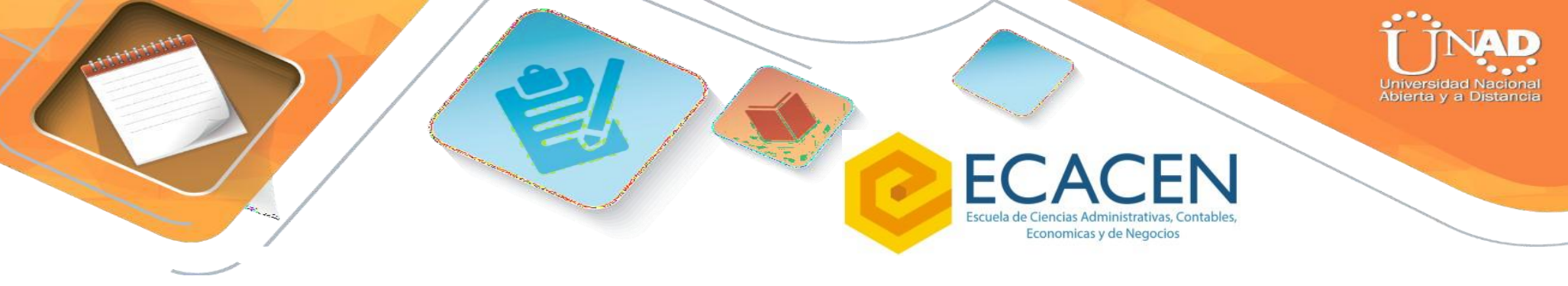

of the labor market. A general theoretical framework is presented on how the 4.0 revolution essentially occurs, and how this should affect traditional education to evolve to new paradigms, generating new demands for the teacher and his pedagogical practice, essentially for the university student and his autonomy to learn in a flexible but meaningful way.

Keywords: Education: 4.0, Industry 4.0, Intelligent Organizations, Revolution 4.0.

\section{INTRODUCCIÓN}

Revolución es el termino usado durante toda la historia para definir cada punto de quiebre de la sociedad que han afectado de manera global las tradiciones construidas con el pasar de los años y que permiten las relaciones, los sistemas productivos, el desarrollo social y la conformación de una cultura determinante en las comunidades.

Revolución 4.0 se entiende como la más reciente de las evoluciones de la sociedad, en la que, cómo sugiere Instituto Politécnico Nacional (2019) se presenta el más actualizado quiebre de la tradicional forma de ser, de producir, de pensar, de concebir, de relacionarse, y de educar. En otras palabras, se dan dos situaciones esenciales. Primero la irrupción (presencia violenta de algo que no estaba) de una nueva mentalidad que demarca el nuevo paradigma futurístico cercano de la sociedad. Por otro lado, la disrupción del estado actual que cancela el futuro trazado estableciendo las nuevas reglas del juego para el cambio, a través de una oferta de futuros diversos.

En tal sentido, la Revolucion 4.0 puede consebirse como la nueva era, que enfoca su esencia en la interconectividad, la automatización y el acceso en tiempo real a los datos (Botella, 2016). Esto, es para garantizar resultados de mayor impacto a nivel económico y social, garantizando la integridad de las personas, los procesos, los equipos y la sociedad en general. Esta revolucion apunta a una nueva esencia en el sector productivo y laboral, por lo que, cómo efecto, no solo la industria se transforma, se requiere una transformación global. Esta ultima idea pone una gran responsabilidad en el sistema educativo tanto básico cómo superior. 


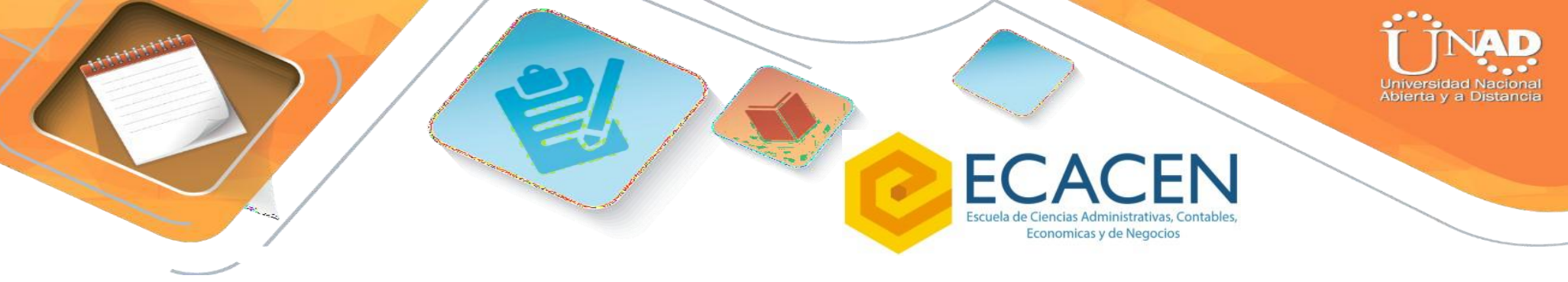

Se requiere una plena identificacion de las competencias que se espera en los estudiantes de educacion superior para migrar a la educacion 4.0 con éxito, para afrontarla y para desarrollarse en funcion de las nuevas entidades productivas, tambien conocidas como Organizaciones Inteligentes.

\section{MARCO TEÓRICO}

Para tener una contextualización teórica de las competencias de educación requeridas en estudiantes de educación superior para migrar a la educación4.0, se considera importante establecer de manera general la relación entre los conceptos de Revolución 4.0, Industria 4.0 y educación 4.0 (ver la figura 1).

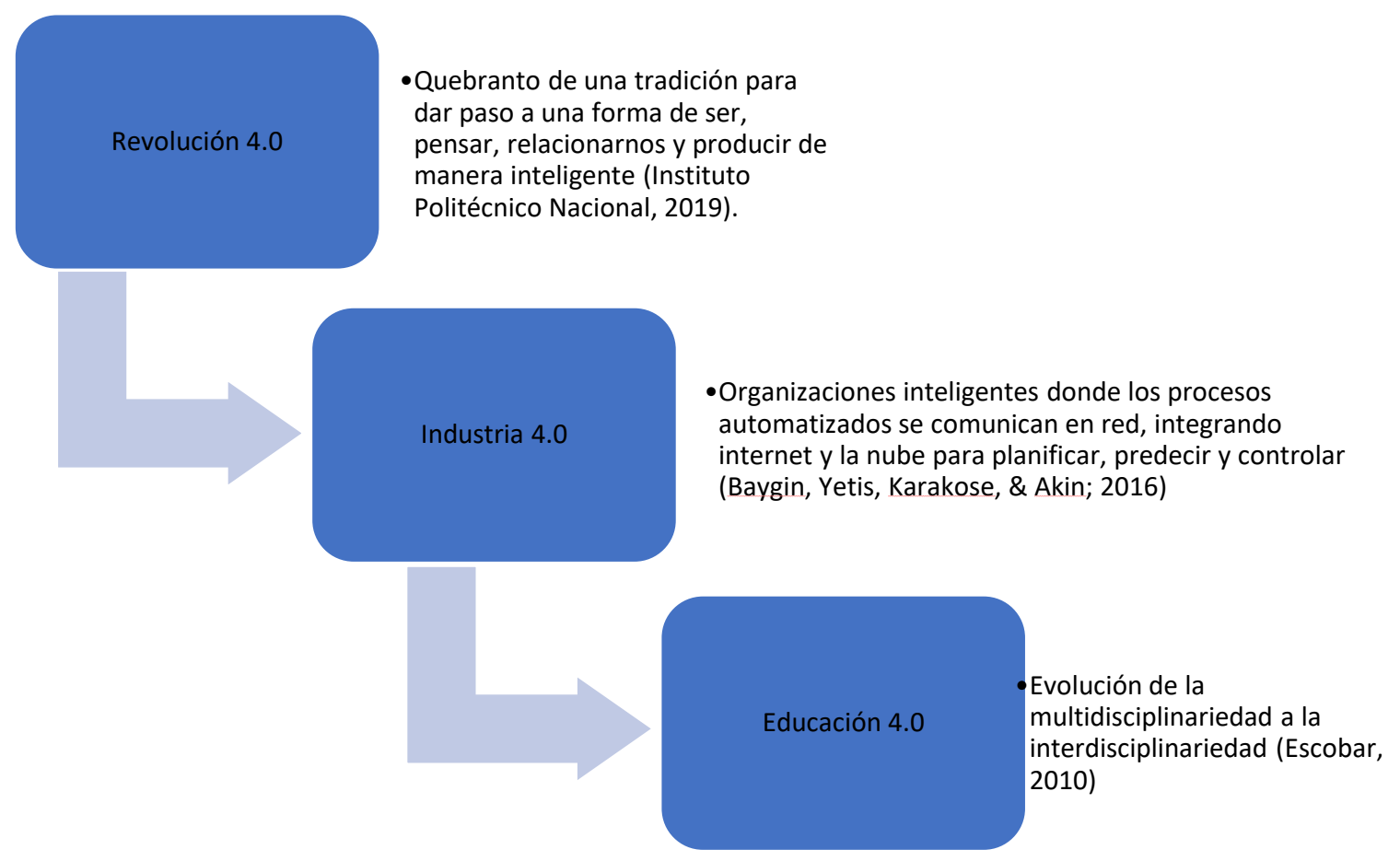

Figura 1.

Nota: Relación que da entre los conceptos más fundamentales que dan paso a la educación 4.0 como una de las bases que garantizan la estabilidad de la Revolución 4.0.

Fuente: elaboración propia 


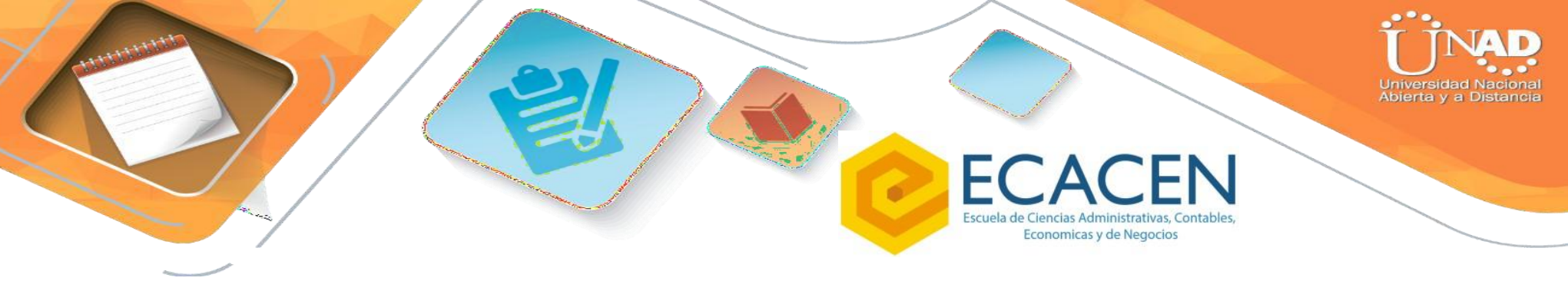

Según Instituto Politécnico Nacional (2019) toda revolución llega para quebrar, transformar e instalar un nuevo orden para hacer las cosas. Se sabe que a nivel global, la Revolución 4.0 ha generado el proceso de romper la tradición, de identificar y satisfacer la necesidad de un cambio de la realidad para ofrecer nuevos y diversos futuros, esto basado en el estudio de los datos en tiempo real. Esta situación, no es sólo en el ambito empresarial, Gauthier \& Méndez(2020)explica que en lo relacionado al ser, esta es una revolución empática, de alguna manera ambidextra,esta ultima cualidad pone a las organizaciones en el siguiente dilema: continuar el negocio o crear el futuro. Tambien esta Cuarta Revolución se caracteriza por tener una memoria selectiva, esto tiene que ver con lo expuesto por Gunther (2013) cuando afirma: "Por cada buena idea que se genera en una empresa, hay por lo menos 100 personas en el resto del mundo desarrollandola la mismo tiempo". Esto quiere decir que se ha dado fin a la ventaja competitiva, siendo realidad la necesidad insaciable de las organizciones de sorprender a sus clientes constantemente, esto es porque lo que puede estar funcionando hoy, mañana sencillamente dejará de ser sensacional. En esta parte se puede retomar la idea de una revolución de esencia disruptiva que ofrece diversos futuros. Lo unico seguro es el cambio.

Todo lo que se ha venido diciendo conecta linealmente con el concepto de Industria 4.0: Fabricas u organizaciones inteligentes, al respecto se tiene el aporte desarrollado por Baygin, Yetis, Karakose, \& Akin (2016)que la definen como la industria integrada por Organizaciones inteligentes donde los procesos automatizados se comunican en red, integrando internet y la nube para planificar, predecir y controlar, esto garantiza mayor acertividad en la proyeccion de los resultados en diferentes escenarios.

De acuerdo a Bauses (2019)una organización inteligente es aquella que cuenta con una estructura organizada para toma de desiciones a nivel innovador frente a problemas que surgen, de manera eficaz y eficiente, basadas en recursos inmateriales como la informacion, el conocimiento, tambien recursos materiales como los financieros y los humanos.

Esta nueva esencia de las organizaciones en general a desarrollado el estudio de un sin número de factores determinantes en la productividad, donde la gestión del conocimiento surge cómo base más fundamental y 


\section{- ECACEN}

donde los procesos de aprendizaje colaborativo son altamente necesarios para lograr los resultados de posicionamiento y sostenibilidad en el mercado. Al respecto se puede mencionar la Quinta Disciplina: cómo impulsar el aprendizaje de la organización inteligente, desarrollada por Senge(2012)en la que aborda la necesidad de ocnstruir organizaciones inteligentes que estén dispuesta al aprendizaje como principal razón de existencia. Para ello el autor plantea las tecnologias o competencias que deben estar presentes en el talento humano de las organizaciones inteligentes, estas son: pensamiento sistémico, dominio personal, modelos mentales, construccion de una visión compartida, aprendizaje en equipo.

Las competencias anteriormente mencionadas vienen a ser las requeridas por cada profesional que ingresa a laborar en una organización inteligente, en tal sentido, la pregutna que surge es ¿Dónde se puede lograr que los profesionales adquieran estas competencias y así sean relevantes para la industria 4.0? la respuesta según Zambrano, Habid, \& Alfaro(2019)está en el sistema de educacion superior, y en tal sentido se empieza a hablar de la Educación 4.0 en el nivel superior. Los autores plantean la necesidad de transformar el sistema educativo, esto implica la transformación y actualización de los curricumlums, las metodologias docentes y las estrategias personales de cada estuditante.

En tal sentido, el presente es un informe parcial en el que se estudian y establecen las nuevas competencias de educación 4.0 que deben tener los estudiantes para garantizar que su formación profesional les permita adquirir las competencias requeridas para enfretarse de manera exitosa en el ambito laboral generado en las organizaciones inteligentes.

\section{METODOLOGÍA}

Se propone el desarrollo de una investigación cualitativa que es definida por Fassio (2018)la investigación que se esfuerzo por el enfasis directo al objeto de estudio, remitiendo obligatoriamente al contexto, tambien estudio la relacion que existe entre la cultura y su conexión con el objeto de investigación, facilitando el diálogo entre los datos y lo teórico. En esta investigación se propuse el objetivo de identificar las competencias requeridas por los estudiantes de educacion superior para migrar a la educacion 4.0 que garantizaría la fomración necesaria para incursionar laboralmente en las industrias inteligentes de la Revolución 4.0. 


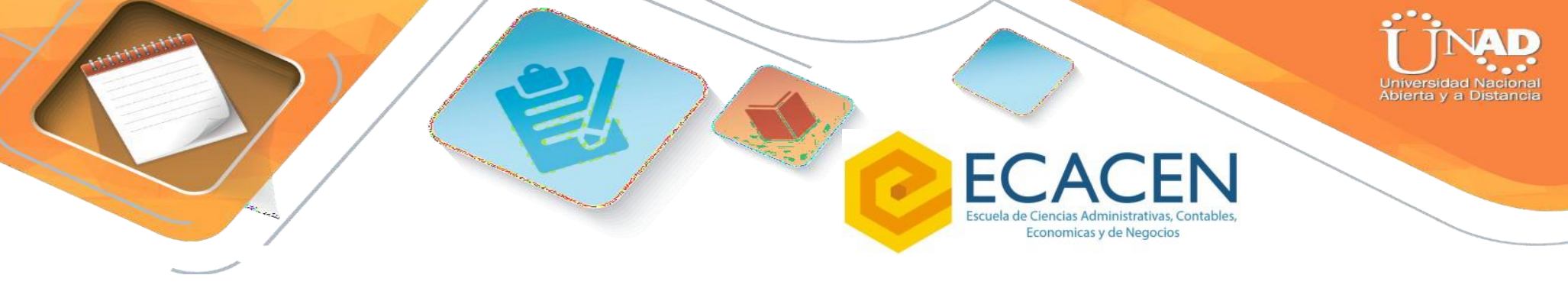

Inicialmente para este informe se hizo una revision de bases de datos sobre investigaciones academicas publicadas que ayudan a la contrucción de un contexto teorico sobre las competencias, esto através de una comparacion muy somera entre las caracteristica de la educación tradicional y la ya mencionada educación 4.0. este informe hace parte de una investigación en curso sobre las competencias de educación 4.0 que deben tener los estudiantes de la UNAD, maxime la condición de esta en la prestación del servicio de educacion superior virtual y a distancia, que justifica como sus estudiantes son mediados principalmente por las tics para llevar a cabo su formación.

\section{RESULTADOS}

Las competencias requeridas para la educación 4.0 en estudiantes de educación superior, son variadas y están relacionadas con las competencias y habilidades que deben tener los profesionales para incursionar en el campo laboral en el marco de la Revolución 4.0. En el presente apartado se presenta de manera general las competencias que deben estructurar la educación 4.0teniendo en cuenta los aportes que se han generado a nivel investigativo en la academia, para ello se presentaran los aportes de diferentes autores que ayudan a establecer tales competencias y la contraparte con la educación tradicional.

Se cree que la digitalización se desarrolló más plenamente en el marco de la más reciente pandemia, sin embargo, esta idea carece de fuerza y argumento toda vez que la era digital inició en el preciso momento que se establecieron las ventajas de los sistemas interconectados, la recolección y monitoreo de datos de en tiempo real, y reduciendo las horas hombre, para el caso de las empresas. La digitalización fue puesta en marcha y la pandemia reciente lo único que hizo fue ratificar su importancia en la sociedad.

Estudios como el de Iglesia (2019)muestran que la Educación 4.0 ha llegado para quedarse, y que ha generado nuevas necesidades a nivel de estrategias y metodologías pedagógicas para la educación y el aprendizaje, lo que lleva directamente a una reformulación de la caja de herramienta docente. En otras palabras, surge la necesidad de que los docentes den un giro a la forma en la que vienen desarrollando los procesos de apropiación del conocimiento por parte de los estudiantes, para ello, es necesario reforzar el uso de herramientas que ya estaban en 


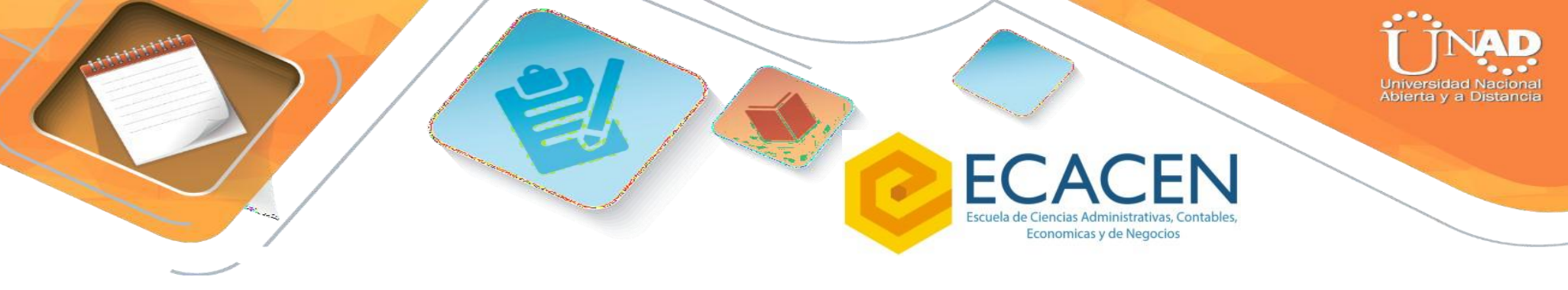

prueba o testeo, sin embargo, ahora es necesario que los docentes se hagan especialistas en el uso de estas herramientas.

Al autor hace enfasis especial en la relevancia que adquiere el enfoque multidisciplinar, la simulación de la realidad como herramienta de aprendizaje, el avance de la incorporación del Internet de las cosas en el aula, la planificación de la gamificación en el aprendizaje, la anexión de los juegos de aprendizaje, etc. Al simular la realidad, algo queda en el conocimiento.

En tal sentido, autores como Olivera, Games, Barragán, \& Cortés (2020)le dan a la educación 4.0 un enfoque netamente económico, puesto que apunta al desarrollo del estudiante como eje estratégico para el desarrollo económico sostenible. Apunta a un mayor numero de herramientas y soluciones tecnológicas que simulan la realidad para aprender a través de la experiencia. En general se concibe como: adaptativo, autorregulado, experimental, activo, interactivo, colaborativo, autodirigido, rizomático, ubicuo, basado en problemas, basado en proyecto.

Por su lado, Zambrano, Habid, \& Alfaro (2019)reinciden en la necediad de que las instituciones se adapten a las nuevas demandas y a la nueva era de la sociedad, el desarrollo y la tecnología. Todo ello implica que la educación superior especialmente se transforme, haciendo cambios importantes no en la esencia sino en los métodos y las herramientas. La educación 4.0 promueve nuevas competencias para los estudiantes por lo que genera la necesidad transformar las aulas, incursionar en mayores niveles de flexibilidad, apelar a la tecnología como aliado estratégico metodológico.Una ilustración más clara de este nuevo paradigma es la introducción de la tecnología en todas las cátedras y currículos, a través de herramientas, formas de relación y método para la producción.

\section{CONCLUSIONES}

La actualidad está caracterizada por un mundo globalmente digital e hiperconectado, la comunicación instantánea ahora es una realidad. Las necesidades de las empresas se han transformado y con ellas lo puestos de trabajo, por ende, la educación también requiere evolucionar, migrar a un carácter más flexible, personalizado, digital y multidisciplinar, ello explica cómo se puede dar una junta entre un chef, un fotógrafo y 


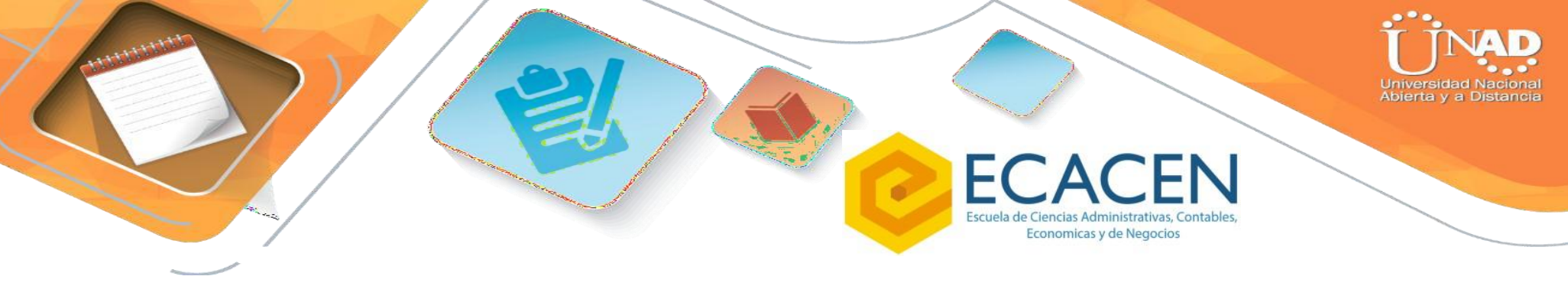

diseñador gráfico, un escritor comercial y un director de marketing y ventas digitales.

La industria 4.0 está caracterizada por tres demandas esenciales que son mayor flexibilidad en la producción, menor tiempo para llevar el producto al mercado y resultados de mayor impacto gracias al análisis de la data. La educación 4.0 requiere satisfacer demandas que permitan que las necesidades de la industria sean cubiertas, estas demandas son: aprendizaje en función e intereses de cada individuo, es decir, más flexible. Procesos educativos personalizados, es decir al ritmo de cada estudiante, su edad y curso. Aprendizaje digital, con feedback garantizado basado en el análisis de datos generado en el proceso (Learning Analytics).

Esto requiere que el docente se ajuste al estilo de aprendizaje de cada estudiante, estudiar los tipos de cerebros y modelos temperamentales pueden ayudar a este reto.Además, escuelas como centro para el desarrollo de talento, eso implica una desaceleración de los programas académicos y un acercamiento más detallado a cada estudiante, desarrollar su talento implica un conocimiento profundo de sus capacidades y potencialidades.

\section{REFERENCIAS}

Bauses, A. (2019). Incidencia de las Organizaciones Inteligentes en la Gestión del Conocimiento. Revista Arbitrada Interdisciplinaria Koinonía, 366-393.

Baygin, M., Yetis, H., Karakose, M., \& Akin, E. (2016). An effect analysis of industry 4.0 to higher education. In 2016 15th International Conference on Information Technology Based Higher Education and Training (ITHET) (pp. 1-4).

Botella, F. (2016). Bienvenidos a la Revolución 4.0. Barcelona: Editorial Alienta.

Fassio, A. (2018). Reflexiones acerca de la metodología cualitativa para el estudio de las organizaciones. Ciencias Administrativas. 
Gauthier, V., \& Méndez, R. (2020). Voces diversas y disuprtivas en tiempos de Revolución 4.0.Universidad del Rosario .

Gunther, R. (2013). The endof Competitive Advantage: How to keep your strategy moving as fast as your business. Estados Unidos : Harvard Business Review Press.

Iglesia, M. (2019). Caja de herramientas 4.0 para el docente en la era de la evaluacion por competencias. Innovación educativa, 93-112.

Instituto Politécnico Nacional. (2019). Innovación educativa: Casos y retos de la educación 4.0. México : ISSN 1665-2673.

Olivera, D., Games, F., Barragán, M., \& Cortés, E. (2020). Educación 4.0, origen para su fundamentación. Contribuciones de la tecnología digital en el desarrollo educativo y social. Educación y Ludica.

Senge, P. (2012). La quinta disciplina: Como impulsar el aprendizaje en la organizacion inteligente. Estados Unidos : Ediciones Granica S.A.

Zambrano, M., Habid, L., \& Alfaro, N. (2019). Educación superior en el contexto de la industria 4.0: Ponencia XV Congreso Nacional de Investigación Educativa. México . 